\title{
Lacrimal Sac Mucocele in a Newborn: A Rare Mimic of Congenital Intranasal Tumor
}

Ayla Gunlemez ${ }^{1 *}$, Selim Oncel ${ }^{2}$, Nagihan Inan ${ }^{3}$, Murat Ozturk ${ }^{4}$, Gulcan Turker ${ }^{5}$ and Funda Corapcioglu ${ }^{6}$

${ }^{1}$ Department of Pediatrics, Division of Neonatology, Kocaeli University, Turkey

${ }^{2}$ Department of Pediatrics, Division of Pediatric Infectious Diseases, Kocaeli University, Turkey

${ }^{3}$ Department of Radiology, Kocaeli University, Turkey

${ }^{4}$ Department of Otorhinolaryngology, Kocaeli University, Turkey

${ }^{5}$ Department of Pediatrics, Division of Neonatology, Kocaeli University, Turkey

${ }^{6}$ Department of Pediatrics, Division of Pediatric Oncology, Kocaeli University, Turkey

\begin{abstract}
Lacrimal sac mucocele, either unilateral or bilateral, occurs when the nasolacrimal duct is obstructed and is a rare anomaly in the newborn period. We presented 1 day old full-term female baby with respiratory distress. She was referred by her primary care provider for an otolaryngologic assesment with the suspicion of choanal atresia. In her nasal endoscopy, a mass with a mucosal lining was filling the right nasal cavity. Ultrasonography showed a submucosal tumorous lesion in the right nasal cavity and MRI demonstrated a unilateal lacrimal sac mucocele. In this case report, it is stressed that lacrimal sac mucocele may manifest only with intranasal mass in the newborn period; which can be misdiagnosed as a tumor. MRI is a valuable tool in its capacity to delineate many details in mucocele and may prevent unnecessary biopsies and other surgical procedures.
\end{abstract}

Keywords: Newborn; Nasal mass; Lacrimal sac mucocele; Magnetic resonance imaging

\section{Introduction}

A newborn, who presents with a midline frontonasal mass, often poses a diagnostic challenge to clinicians [1-3]. A congenital nasal mass usually manifests itself with respiratory distress, nasal obstruction, or as a protruding mass in the newborn period. Determination of presence of any connections between the mass and normal anatomic structure with imaging techniques is crucial in the management [4-6]. The differential diagnosis include encephalocele, nasal glioma, epidermoid cyst, dermoid cyst, hemangioma and embryonic tumors such as rhabdomyosarcoma, teratoma, and neuroblastoma [1,7,8]. Lacrimal sac mucocele, on the other hand, usually presents itself as a blueish medial cantal mass, epiphora, overflow of tears, or rarely dispnea $[1,4,5,9]$. Magnetic Resonance Imaging (MRI) is the imaging modality of choice in assessing frontonasal masses in neonates due to its capacity to reveal developmental anomalies among other problems [1].

In the presented patient, rhinoscopy, which was performed in the absence of overflow of tears in order to find out the cause underlying dispnea showed an intranasal mass. The patient was diagnosed afterwards as lacrimal sac mucocele, thanks to the typical clinic and MRI findings. It should be kept in mind that a nasal mass can be the sole presentation of lacrimal sac mucocele, especially in newborns.

\section{Case Report}

The patient was a full-term female, born following an uncomplicated pregnancy and vaginal delivery. Her nasal and oral secretions were reported to have been aspirated immediately after delivery. Her parents have realized that she had been having difficulty in breathing and a change of color of her skin to purple while feeding. In the postnatal seventh hour, she was referred by her primary care provider for an otolaryngologic assesment with the suspicion of choanal atresia. In nasal endoscopy, a mass with a mucosal lining was filling the right nasal cavity, showing no change of color, and causing the septum to deviate to right was seen (Figure 1).

In the neonatology and pediatric oncology consultation, a $6 \mathrm{~F}$ feeding tube could be passed through both nostrils. Mild tachypnea, noisy breathing and cyanosis while feeding were noted. There was no other pathological

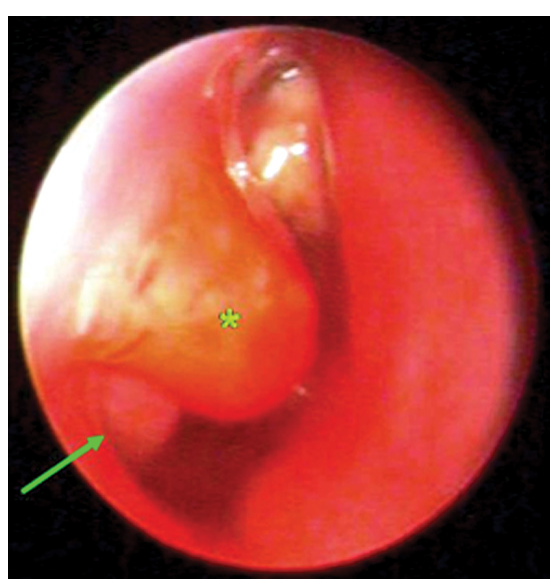

Figure 1: Endoscopic examination showing a bluish cystic mass beneath inferior turbinate (The arrow indicates the mucocele, * indicates middle turbinate).

finding in physical examinations and laboratory tests, including complete blood count, serum biochemistries, and blood gas analysis, urinalysis and chest $\mathrm{x}$-ray. She needed $30 \%$ oxygen with head box only while feeding on day 1. Ultrasonography showed a submucosal tumorous lesion in the right nasal cavity. Since association of this lesion with the anatomical structures was not properly determined, MRI utilizing precontrast axial T2-weighted Turbo-Spin Echo (TSE) (Figure 2a) and coronal short tau inversion recovery

*Corresponding author: Ayla Gunlemez, Department of Pediatrics, Division of Neonatology, Kocaeli University, Turkey, Tel: +90 26230310 00; E-mail: aylagunlemez@yahoo.com

Received August 24, 2015; Accepted September 10, 2015; Published September 17, 2015

Citation: Gunlemez A, Oncel S, Inan N, Ozturk M, Turker G (2015) Lacrimal Sac Mucocele in a Newborn: A Rare Mimic of Congenital Intranasal Tumor. J Clin Case Rep 5: 600. doi:10.4172/2165-7920.1000600

Copyright: (C) 2015 Gunlemez A, et al. This is an open-access article distributed under the terms of the Creative Commons Attribution License, which permits unrestricted use, distribution, and reproduction in any medium, provided the original author and source are credited. 

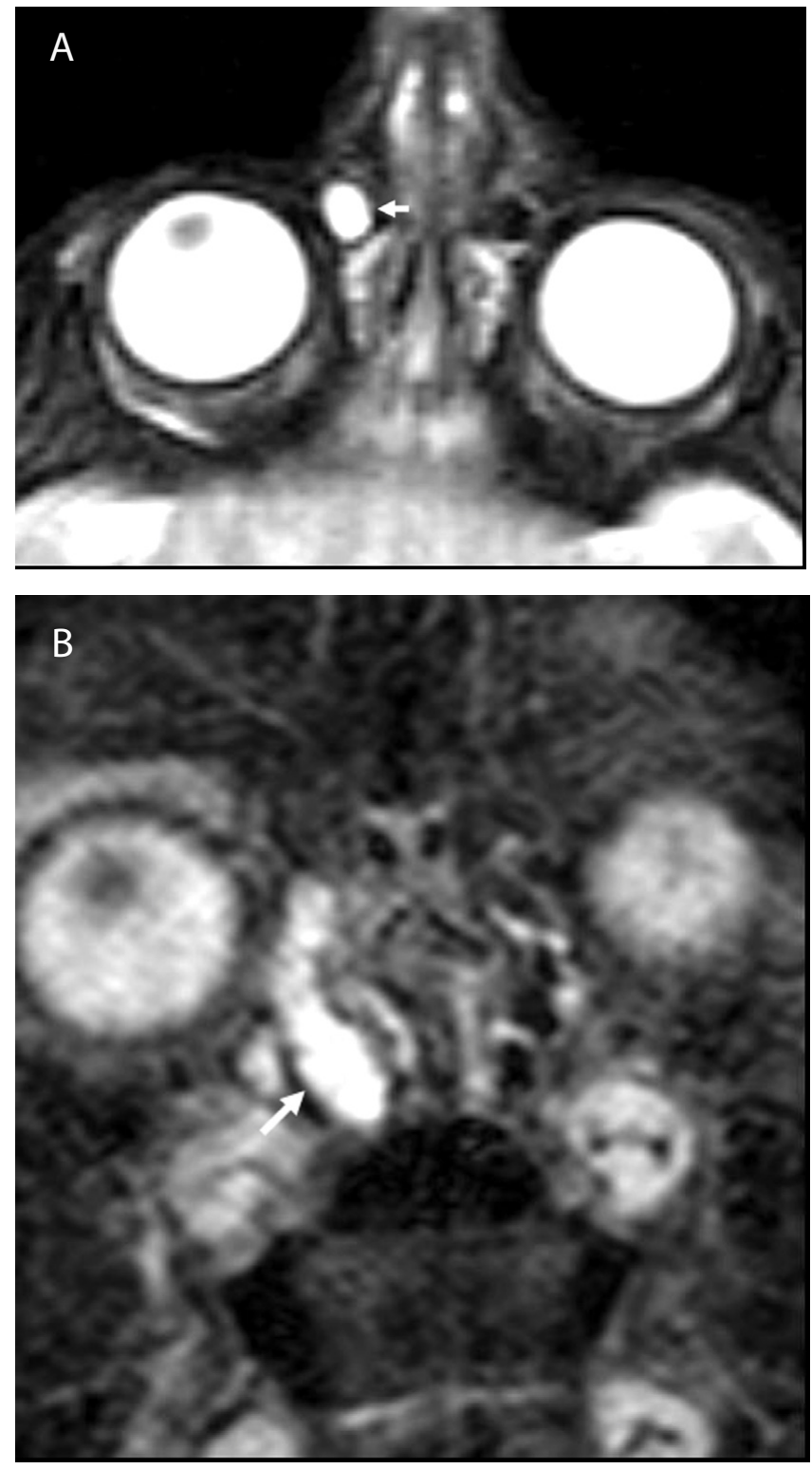

Figure 2: Fat suppressed axial T2-weighted turbo-spin echo (TSE). (a) and coronal (b) short tau inversion recovery (STIR) images showing hyperintense, cystic dilatation of nasolacrimal duct (white arrow).

(STIR) (Figure 2b) with a slice thickness of $3 \mathrm{~mm}$ and an intersection gap of $0.3 \mathrm{~mm}$, was carried out, which demonstrated a unilateal lacrimal sac mucocele extending from the inferomedial canthus to the inferior nasal cavity on the right. This dilatation was hypointense on T1-weighted and homogeneously prominent hyperintense on T2-weighted TSE and STIR images (Figure 2a, Figure 2b; white arrow) with no remarkable contrast enhancement. Because of the absence of any associations of the mass lesion with the anterior cranial fossa and the brain, the intactness of the frontonasal bony structures, and the intensity characteristics; anterior neuroporous pathologies, such as nasal glioma, epidermoid cyst, dermoid cyst, together with hemangioma and solid embryonal tumors have been ruled out. Experiencing neither difficulty breathing nor an infection in the nasolacrimal sac during the course of her three-month follow-up period, our patient needed no therapy, either medical or surgical, apart from regular gentle massaging of her right nasal region.

\section{Discussion}

Tumors of the nasal cavity are rarely seen in pediatric population. Definitive histopathologic evaluation is needed for diagnosis [6]. The patient's age has a leading role in clinical approach and presumptive diagnosis. Contrary to epithelial neoplasia in adults, rhabdomyosarcoma and non-rhabdoid soft tissue sarcomas are the most frequently encountered malignant tumors in patients less than 18 years of age [1012]. Unfortunately, there are delays in diagnosis and treatment in the pediatric population since symptoms are non-specific [6]. Differential diagnosis of nasal masses in the newborn period includes malignant tumors, such as rhabdomyosarcoma, neuroblastoma and teratoma. Benign pathologies, that may manifest themselves with an intranasal mass, are nasal glioma, encephalocele, hemangioma, epidermoid cyst, dermoid cyst, polyp, and lacrimal sac mucocele $[1,2,8,13,14]$. All these malignant and benign pathologies manifest clinically as signs of midfacial or canthal mass, nasal obstruction, and protruding lesion in endoscopy [1]. Nasal obstruction is an important cause of respiratory distress, particularly in the neonatal period [4]. Although the most common cause of nasal obstruction in neonates is choanal atresia/ stenosis, congenital cysts, hamartomas, hemangiomas, encephalocele, and congenital neoplasms should also be considered in differential diagnosis $[4,15]$. Lacrimal sac mucocele, either unilateral or bilateral, occur when the nasolacrimal pathway is obstructed and it is a rare anomaly in the newborn [2,5]. The reason is usually an impaired canalization at the distal end of the nasolacrimal duct with a residual membrane between the duct and the nasal cavity. Failure of canalization occurs most commonly at the nasal portion of the duct. Obstruction can be complete or incomplete. The canal may expand and take the form of a sac as fluid accumulates [3]. The swelling of lacrimal sac is observed at birth; but newborns diagnosed in the prenatal ultrasonographic screening have also been reported [3,5,9,16-20]. Prolapse or expansion of the mucocele into nose may lead to respiratory distress and diffulty in feeding, as newborns are preferential nose breathers [16].

The clinical picture, which usually consists of a medially-placed blue-grey-colored canthal mass, epiphora, overflow of tears, and mucoid ocular discharge may become complicated with dacryocystitis, preseptal/orbital cellulitis, or even sepsis $[2,4,15,21,22]$. In the presented case, there was no sign of lacrimal sac mucocele except for respiratory distress. Still very rare cases without canthal mass or epiphora have been reported [4,23]. About half of lacrimal sac mucoceles are bilateral, in $70 \%$ of which, signs of respiratory distress are observed $[2,16,24]$.

Nasal examination should not be neglected in a newborn with respiratory distress $[3,16,22]$. Neverthless, if other physical signs are inconsistent with mucocele, as was the case in our patient, diagnosis can be supported by radiologic imaging. Ultrasound is a simple and non-invasive method for reliably distinguishing mucocele from solid masses; but it may not provide a definite differentiation from cystic lesions, such as dermoid cyst, epidermoid cyst, encephalocele, and hemangioma. Computed tomography has the advantage of detecting bone changes involving the bony portion of the nasolacrimal canal; but MRI seems more advantageous in newborns because of its superior delineation of unusual morphology and maturation of the frontonasal region and absence of radiation exposure [15,25]. Another advantage of MRI is the in-utero applicability.

A case of lacrimal sac mucocele with intranasal extension has been described by MRI characteristics. Magnetic resonance imaging can reveal the cyst content, allowing multiplan imaging without the need for manipulation of the patient. The signal intensity of cyst content was helpful in characterizing of the lesion, allowing us to differentiate 
Citation: Gunlemez A, Oncel S, Inan N, Ozturk M, Turker G (2015) Lacrimal Sac Mucocele in a Newborn: A Rare Mimic of Congenital Intranasal Tumor. J Clin Case Rep 5: 600. doi:10.4172/2165-7920.1000600

it from similar soft-tissue pathologies, in particular, dermoid cyst. The low signal on T1- and high signal on T2-weighted images were consistent with the mucoid or proteinaceous material, a usual finding in a mucocele [15].

Determination of the presence of a connection between frontonasal mass and the anterior cranial fossa is crucial in the imaging assessment for encephaloceles. Protrusion of meninges, brain, and bony dehiscence at the frontonasal junction is diagnostic for cephaloceles [1]. Nasal glioma is also among the mass lesions arising in this region. Along with its expansile solid characteristic, its inability to hold contrast medium is typical. Contrast enhacement is observed in other solid tumors, such as rhabdomiosarcoma. The treatment of congenital lacrimal sac mucocele is controversial. It usually resolves spontaneously and requires surgical intervention only rarely. In newborns with severe respiratory distress, surgical measures, such as probing with decompression into nose, baloon catheter dacryocystoplasty, silicone intubation, and marsupialization may be recommended although in asymptomatic patients, all that is necessary is massaging, and antibiotics for infections [3,22]. Resolution of the mucocele can take place with expectant management [26].

Perhaps the temporary respiratory distress in our patient was due to the mucosal edema and accumulation of nasal secretions caused by the aspiration that was performed just after the delivery.

In this case report, it is stressed that lacrimal sac mucocele may manifest only with intranasal mass in the newborn period; which can be misdiagnosed as a tumour. MRI is a valuable tool in its capacity to delineate many details in mucocele and may prevent unnecessary biopsies and other surgical procedures.

\section{References}

1. Hedlund G (2006) Congenital frontonasal masses: developmental anatomy, malformations and MR imaging. Pediatr Radiol 36: 647-662.

2. Tsai YS, Huang JK (2006) Neonatal nasal obstruction caused by bilateral dacrocystoceles. Pediatr Radiol 36: 1221.

3. Levin AV, Wyngnanski-Jaffe T, Forte V, Buckwalter JA, Buncic JR (2003) Nasal endoscopy in the treatment of congenital lacrimal sac mucoceles. Int J Pediatr Otorhinolaryngol 67: 255-261

4. VarnelL H, Friedman NR, Shea C, Jones MD (2003) An unusual cause of neonatal respiratory distress. J Perinatology 23: 688-690.

5. Teixeira CC, Dias RJ, Falcao-Reis FM, Santos M (2005) Congenital dacryocystocele with intranasal extension. Eur J Ophthalmol 15: 126-128.

6. Benoit MM, Bhattacharyya N, Faquin W, Cunningham M (2008) Cancer of the nasal cavity in the pediatric population. Pediatrics 121: 141-145.

7. Biasio P. De, Scarso E, Prefumo F (2006) Prenatal diagnosis of a nasal glioma in the mid trimester. Ultrasound Obstet Gynecol 27: 571-573.

8. Gnanaraj L, Skibell BC, Coret-Simon J (2005) Massive congenital orbital teratoma. Ophthal Plast Reconstr Surg 21: 445-447.
9. Goldberg H, Sebire NJ, Holwell D, Hill S (2000) Prenatal diagnosis of bilateral dacrocystoceles. Ultrasound Obstet Gynecol 87: 448-449.

10. Herrmann B, Sotelo-Avila C, Eisenbeis M (2003) Pediatric sinonasal rhabdomyosarcoma: three cases and a review of the literature. Am J Otolaryngol 24: 174-180.

11. Eich H, Hero B, Staar S, Micke O, Seegenschmiedt H, et al. (2003) Multimodality therapy including radiotherapy and chemotherapy improves event-free survival in stage C esthesioneuroblastoma. Strahlenther Onkol 179: 233-240.

12. Bhattacharyya N (2002) Cancer of the nasal cavity: survival and factors influencing prognosis. Arch Otolaryngol Head Neck Surg 128: 1079-1083.

13. Corapçıoglu F, Ozek MM, Sav A, Uren D (2006) Congenital pineoblastoma and parameningeal rhabdomyosarcoma: concurrent two embryonal tumors in young infant. Childs Nerv Syst 22: 533-538.

14. Mathew PJ, Gombar KK (2005) Neonatal nasal polypectomyconsegence of nasal packing. Acta Anaesthesiol Scand 50: 522 Farrer RS, Mohammed TL, Hahn FJ (2003) MRI of childhood dacryocystocele. Neuroradiology 45: 259-261.

15. Yee SW, Selbert RW, Bower CM, Glasier CM (1994) Congenital nasolacrimal duct mucocele: a cause of respiratory distress. Int J Pediatr Otorhinolaryngol 29: 151-158.

16. Alper C, Chan K, Hill L, Cheveney P (1994) Antenatal diagnosis of a congenital nasolacrimal duct cyst by ultrasonography: a case. Report Prenatal Diagnosis 14: $623-626$.

17. Salvetat ML, D'Ottavio G, Pensiero S, Vinciquerra A, Perisutti P (1999) Prenatal sonographic detecti,on of a bilateral dacrocystocele. J Pediatr Ophthamol Strabismus 36: 295-297.

18. Sharony R, Raz J, Aviram R (1999) Prenatal diagnosis of dacrocystocele: a possible marker for syndromes. Ultrasound Obstet Gynecol 14: s71-73.

19. A, Amin N, Cornell C (1997) Antenatal sonographic diagnosis of dacrocystocele. J Maternal-Fetal Med 6: 273-275.

20. Helper KM, Woodson GE, Kearns DB (1995) Respiratory distress in the neonate: Sequela of a congenital dacryocystocele. Arch Otolaryngol Head Neck Surg 121: 1423-1425.

21. Becker BB (2006) The Treatment of Congenital Dacryocystocele. Am J Ophthalmol 142: 835-838.

22. Right PD, Hubbell RN, Lawlor PP (1993) Respiratory distress associated with bilateral nasolacrimal duct cysts. Int J Pediatr Otorhinolaryngol 26: 199-203.

23. Paysee EA, Coats DK, Bernstein JM, Go C, de Jong AL (2000) Management and complications of congenital dacryocele with concurrent intranasal mucocele. J Aapos: Am Assoc Pediatr Ophthalmol Strabismus 4: 46-53.

24. Bianchini E, Zirpoli S, Raghini A (2004) Magnetic resonance imaging diagnosis of dacryocysocele. J Comput Assist Tomogr 28: 422-427.

25. Schnall BM, Christian CJ (1996) Conservative treatment of congenital dacryocele. J Pediatr Ophthalmol Strabismus 33: 219-221. 